\title{
Short communication Current and future choices in endocrine therapy
} Matthew Ellis

Washington University School of Medicine, Division of Oncology, South Euclid Ave, St Louis, Missouri 63119, USA

Corresponding author: Matthew Ellis, mellis@wustl.edu

Published: 20 December 2007

This article is online at $\mathrm{http}: / /$ breast-cancer-research.com/content/9/S2/S15 (c) 2007 BioMed Central Ltd

A typical article on choices in endocrine therapy for breast cancer generally focuses on a discussion of the use of an adjuvant aromatase inhibitor versus tamoxifen, a comparison between aromatase inhibitors, the value of ovarian suppression, the use of the steroidal oestrogen fulvestrant, or possible roles for oestrogens and progestins as third-line treatment in the management of advanced disease [1]. To this menu one can now add the use of raloxifene as opposed to tamoxifen as a chemoprevention agent in women at high risk for the disease [2]. All of these options have been examined in randomized clinical trials, but although positive results have been observed, no recent studies have demonstrated an impact on the disease that is close to that seen when tamoxifen was compared with observation in the worldwide overview of randomized tamoxifen trials in breast cancer [3]. One can therefore reasonably conclude that a major phase of drug development in breast cancer endocrine therapy is drawing to a close. Although there may be advances in oestrogen receptor (ER) targeting at the margins, no major breakthrough is likely with the endocrine studies that remain outstanding. The debate is therefore beginning to refocus anew on how we might influence the near constant relapse rate that continues more than a decade after a diagnosis of ER-positive disease, despite the application of our best ER targeting strategies.

So why is it that endocrine therapy does not cure all cases of ER-positive breast cancer, and what are the research choices that we should be making in order to design definitive clinical trials that address this critical problem? The conventional answer to this question is that ER-positive breast cancer is a spectrum of tumour types with different patterns of somatic mutations that influence ER function and reduce the efficacy of endocrine agents [4]. The best support for this hypothesis is the now definitive conclusion that HER2 gene amplification reduces efficacy of endocrine approaches to ER-positive breast cancer. In a variety of contexts, including endocrine therapy for advanced disease, the adjuvant endocrine setting and the neoadjuvant endocrine setting, the consistent pattern of evidence demonstrates that ER-positive, HER2-positive breast cancer does poorly in comparison with ER-positive,
Breast Cancer Research 2007, 9(Suppl 2):S15 (doi:10.1186/bcr1813)

HER2-negative disease [5]. Furthermore, treatment with trastuzumab dramatically reduces the relapse rate for these patients when combined with chemotherapy. So where will we find the next example of a somatic mutation that can be targeted in the remaining cases of poor prognosis ERpositive, HER2-negative disease? The answer to this question must evolve from a study of the breast cancer genome using techniques such as array comparative genomic hybridization and high-throughput sequencing [4].

Examples of other receptor tyrosine kinase amplification events that might be relevant to the problem of endocrine therapy resistance include fibroblast growth factor receptor (FGFR) 1 amplification, which appears to be relatively common in ER-positive disease (frequency about 10\%) and insulin-like growth factor 1 receptor (IGF1R) amplification, which is less common (frequency $<5 \%$ ), but it is of considerable interest because of the new generation of therapeutic IGF1R monoclonal antibodies. Downstream of the receptor tyrosine kinases include gain-of-function mutations in the $\alpha$ catalytic subunit of phosphoinositol-3kinase (PI3K), a AKT1 pleckstrin homology domain mutation, loss of PTEN and amplification of RPS6KB1. As the clinical phenotype of these mutations becomes clear, we can assess their potential for pharmacological targeting. For example, FGFR1 gene amplification was recently shown to be associated with a poor prognosis [6]. In contrast, PIK3CA mutation may be associated with good prognosis and therefore is perhaps less valuable as a target [7]. Nonetheless, it would appear likely that PIK3CA mutations will be present in patients with advanced disease, and therefore direct inhibitors of PI3K might be of some value in this setting.

At Washington University, and in other laboratories around the world, we are beginning to develop a map of these genomic changes within the context of several neoadjuvant aromatase inhibitor trials in order to gain insights into the effects of these mutations on sensitivity to endocrine therapy. We term this project 'The Luminal Breast Cancer Genome Atlas' [4]. The neoadjuvant approach is distinct from efforts 
that simply correlate baseline mutations with relapse-free survival, because relapse data in adjuvant endocrine therapy combine the effect of a mutation/amplification on both prognosis and prediction. In contrast, in the neoadjuvant setting, one can purely look at the impact of any given mutation on endocrine therapy response as distinct from prognosis. The neoadjuvant data will therefore give rise to profiles that are different to, but complementary with, tumour profiles that are trained on relapse data. In addition, an understanding of the effects of mutations in the neoadjuvant setting sets the scene (produces data useful for a power calculation) for trials that partner endocrine agents with new signal transduction agents, as and when these agents become available for study in the early disease setting. We would strenuously argue that all potential pairings of an aromatase inhibitor with a new signaling agent should be tested in a proof-of-principle study in the neoadjuvant setting before a large-scale adjuvant clinical trial is undertaken [8]. A comparison between the outcome of the P024 trial (which compared letrozole and tamoxifen in the neoadjuvant setting in about 300 patients) and that of the BIG (Breast International Group) 1-98 trial (which conducted the same comparison in about 8,000 patients) [9] shows that the neoadjuvant study successfully predicted the superiority of letrozole 5 years before the adjuvant result with $3 \%$ of the study population. The on time accrual of the ongoing Z1031 trial (a neoadjuvant study that compares letrozole, anastrozole and exemestane conducted by the American College of Surgeons Oncology Group) underscores that these studies can successfully be conducted in cancer clinical trial cooperative groups, despite protocol requirements for fresh tissue biopsies at diagnosis and surgery.

The hope for the future is that the next phase of endocrine therapy choices will not be based on small incremental benefits in a broad spectrum of ER-positive tumours, but large benefits in sometimes rather small subgroups of patients defined though the presence of a targetable somatic mutation that interferes with endocrine therapy responsiveness. Improvements in the treatment of patients with ERpositive, HER2-positive disease demonstrate that these advances are possible, and we now have new leads from genomic profiling that we should be able to take advantage of in the near future.

\section{Acknowledgement}

This article has been published as part of Breast Cancer Research Volume 9 Supplement 2, 2007: Controversies in Breast Cancer. The full contents of the supplement are available online at http://breastcancer-research.com/supplements/9/S2.

\section{References}

1. Ellis MJ, Hayes DF, Lippman ML: Treatment of Metastatic Breast Cancer: Diseases of the Breast, 3rd ed. Philadelphia, PA: Lippincott Williams and Wilkins; 2004.

2. Bevers TB: The STAR Trial: evidence for raloxifene as a breast cancer risk reduction agent for postmenopausal women. J Natl Compr Canc Netw 2007, 5:719-724.

3. Early Breast Cancer Trialists' Collaborative Group: Tamoxifen for early breast cancer: an overview of the randomised trials. Lancet 1998, 351:1451-1467.

4. Ellis MJ, Dixon M, Dowsett M, Nagarajan R, Mardis E: A luminal breast cancer genome atlas: progress and barriers. J Steroid Biochem Mol Biol 2007, 106:125-129.

5. Ellis MJ, Tao Y, Young O, White S, Proia AD, Murray J, Renshaw L, Faratian D, Thomas J, Dowsett M, et al.: Estrogen-independent proliferation is present in estrogen-receptor HER2-positive primary breast cancer after neoadjuvant letrozole. $J$ Clin Oncol 2006, 24:3019-3025.

6. Elbauomy Elsheikh S, Green AR, Lambros MB, Turner NC, Grainge MJ, Powe D, Ellis IO, Reis-Filho JS: FGFR1 amplification in breast carcinomas: a chromogenic in situ hybridisation analysis. Breast Cancer Res 2007, 9:R23.

7. Pérez-Tenorio G, Alkhori L, Olsson B, Waltersson MA, Nordenskjöld B, Rutqvist LE, Skoog L, Stål O: PIK3CA mutations and PTEN loss correlate with similar prognostic factors and are not mutually exclusive in breast cancer. Clin Cancer Res 2007, 13:3577-3584.

8. Ellis MJ: Neoadjuvant endocrine therapy as a drug development strategy. Clin Cancer Res 2004, 10:391S-395S.

9. Ellis MJ, Coop A, Singh B, Mauriac L, Llombert-Cussac A, Jänicke $\mathrm{F}$, Miller WR, Evans DB, Dugan M, Brady C, et al.: Letrozole is more effective neoadjuvant endocrine therapy than tamoxifen for ErbB-1- and/or ErbB-2-positive, estrogen receptor-positive primary breast cancer: evidence from a phase III randomized trial. J Clin Oncol 2001, 19:3808-3816. 